\title{
Características da fonetografia em mulheres com equilíbrio dentofacial
}

\author{
Characteristics of phonetography in women with \\ dentofacial balance
}

\author{
Cibele Carmello Santos ${ }^{1}$, Cláudia Tiemi Mituuti² ${ }^{2}$ Giédre Berretin-Felix ${ }^{3}$, Lídia Cristina da Silva Teles $^{4}$
}

\begin{abstract}
RESUMO
Objetivo: Caracterizar o perfil da extensão vocal de mulheres com equilíbrio dentofacial, por meio da fonetografia. Métodos: Fizeram parte desse estudo 22 estudantes do gênero feminino, com idades entre 18 e 29 anos ( $\bar{X}=20 \pm 2$ anos), falantes do Português Brasileiro, sem alteração ou treino vocal, que apresentaram tipo de face padrão I e harmonia dento-oclusal. Foi aplicado um formulário específico de auto-resposta e também foi realizada avaliação antropométrica da face, avaliação dento-oclusal, exame da fonetografia e análise acústica da frequência fundamental habitual da voz das participantes, realizados no período pósmenstrual. Resultados: Os valores encontrados para os diferentes parâmetros analisados foram: frequência fundamental mínima: $\bar{X}=167,32 \pm 22,27 \mathrm{~Hz}$ ou $\bar{X}=40,09 \pm 2,37$ st, frequência fundamental máxima: $\bar{X}=908,45 \pm 210,93 \mathrm{~Hz}$ ou $\bar{X}=69,09 \pm 4,13$ st, extensão vocal: $\bar{X}=29,00 \pm 3,85$ st, intensidade mínima: $\bar{X}=69,32 \pm 4,07 \mathrm{~dB}$, intensidade máxima: $\bar{X}=113,14 \pm 6,10 \mathrm{~dB}$, extensão dinâmica máxima: $\bar{X}=3,91 \pm 5,71 \mathrm{~dB}$, área do fonetograma: $\overline{\mathrm{X}}=709,50 \pm 182,92 \mathrm{~dB}$.st ou $\overline{\mathrm{X}}=31,93 \pm 8,23 \mathrm{~cm}^{2}$, frequência fundamental habitual da vogal "a": $\bar{X}=211,00 \pm 22,55 \mathrm{~Hz}$. Conclusão: No presente estudo foram obtidas as intensidades mínimas e máximas para as distintas frequências alcançadas pelas participantes, bem como a frequência fundamental habitual, estabelecendo os valores de referência quanto ao perfil da extensão vocal de mulheres brasileiras sem alterações vocais e com equilíbrio dentofacial.
\end{abstract}

Descritores: Voz/fisiologia; Fonação/fisiologia; Qualidade da voz; Acústica da fala; Face; Sistema estomatognático

\section{INTRODUÇÃO}

A fonetografia é um exame que representa graficamente a intensidade mínima e máxima da emissão da voz para diferentes frequências, a partir da grave para a mais aguda. É, portanto a representação da extensão vocal do indivíduo. Ela é amplamente utilizada como um método de análise acústica de voz, pois explora e documenta as extremas capacidades ou

Trabalho realizado no Curso de Fonoaudiologia da Faculdade de Odontologia de Bauru da Universidade de São Paulo - USP - Bauru (SP), Brasil.

(1) Pós-graduanda (Mestrado) em Fonoaudiologia do Departamento de Fonoaudiologia da Faculdade de Odontologia de Bauru da Universidade de São Paulo - USP - Bauru (SP), Brasil.

(2) Pós-graduanda (Mestrado) em Fonoaudiologia do Departamento de Fonoaudiologia da Faculdade de Odontologia de Bauru da Universidade de São Paulo - USP - Bauru (SP), Brasil.

(3) Doutora, Professora Adjunto do Departamento de Fonoaudiologia da Faculdade de Odontologia de Bauru da Universidade de São Paulo - USP Bauru (SP), Brasil.

(4) Doutora, Professora Adjunto do Departamento de Fonoaudiologia da Faculdade de Odontologia de Bauru da Universidade de São Paulo - USP Bauru (SP), Brasil.

Endereço para correspondência: Lídia Cristina da Silva Teles. Al. Dr. Octávio Pinheiro Brizolla, 9/75, Vila Universitária, Bauru (SP), Brasil, CEP:

17012-901. E-mail: lidiactm@usp.br

Recebido em: 2/7/2009; Aceito em: 23/12/2009 possibilidades vocais por meio da elaboração de um gráfico que mostra a intensidade dinâmica, em função da faixa de da frequência fundamental ${ }^{(1)}$.

Ela pode ser utilizada como ferramenta para comparações intra e intersujeitos, permitindo acompanhar a evolução da voz durante o crescimento, o envelhecimento e o tratamento ou treino vocal. Possibilita, ainda, caracterizar a voz de cantores de acordo com o gênero, bem como as limitações vocais decorrentes de distúrbios ${ }^{(2-4)}$.

Sabe-se que a qualidade vocal é modulada pelas cavidades ressonantais da face, porém, são escassos na literatura trabalhos que relacionem as condições morfológicas orofaciais e a função fonatória. Em cantores de ópera, as dimensões do trato vocal foram diretamente relacionadas às características da qualidade vocal e dos formantes ${ }^{(5)}$. Nos indivíduos com face curta ocorre um deslocamento da frequência dos formantes em direção aos agudos, enquanto nos casos de face longa há tendência de deslocamento aos graves ${ }^{(6)}$.

O efeito da idade sobre as características vocais foi estudado e comparado em meninos e meninas entre seis e 11 anos, demonstrando diferença entre os gêneros ${ }^{(7)}$. Especificamente nas mulheres, ocorre uma drástica diminuição da frequência fundamental da fala com o envelhecimento, tendo relação com os efeitos hormonais associados com a menopausa. A dimi- 
nuição da secreção do hormônio estrógeno pós-menopausa induz a alterações morfológicas na laringe, levando a uma diminuição nas frequências fundamentais ${ }^{(8)}$. Na literatura foram encontrados trabalhos que analisaram o perfil da extensão vocal de mulheres brasileiras com vozes normais por meio da fonetografia, sendo que um deles avaliou mulheres entre $41 \mathrm{e}$ $60 \operatorname{anos}^{(9)}$, e outro de 17 a 31 anos $^{(10)}$; porém tais estudos não consideraram a condição dentofacial das participantes.

Tendo em vista que a fonetografia é um importante auxiliar na avaliação da voz, e que as condições da face podem influenciar nas características da qualidade vocal, o objetivo do presente estudo foi verificar o perfil da extensão vocal em mulheres falantes do Português Brasileiro com condições craniofaciais equilibradas e sem distúrbios vocais.

\section{MÉTODOS}

Este trabalho cumpriu todas as normas éticas preconizadas para pesquisas envolvendo seres humanos, tendo recebido aprovação do Comitê de Ética, Ensino e Pesquisa da Faculdade de Odontologia de Bauru da Universidade de São Paulo sob protocolo $n^{\circ} 40 / 2005$. Esta pesquisa foi financiada por projeto PIBIC/CNPq de número de protocolo 024/2005.

Participaram deste estudo 22 estudantes do gênero feminino, com idades entre 18 e 29 anos (média 20 anos e dois meses \pm dois anos e três meses). Foram considerados critérios para inclusão: apresentar tipo facial médio com os terços médio e inferior em equilíbrio; comprimento adequado dos lábios superior e inferior, tipo facial padrão $I^{(11)}$; relação do primeiro molar permanente em classe $\mathrm{I}^{(12)}$, trespasse vertical e horizontal com medidas entre 1 e 4 mm ${ }^{(13)}$; não apresentar alterações vocais; não ter comprometimentos sindrômicos ou neurológicos relacionados à fala ou linguagem expressiva ou receptiva; ser falante nativo do Português Brasileiro; não ter sido submetida a cirurgias laríngeas; não ter treino vocal prévio; não ter queixa auditiva; não ser etilista; não ser e nunca ter sido fumante; apresentar bom estado de saúde geral; ausência de alterações laríngeas ou respiratórias e não estar no período menstrual ou pré-menstrual no dia do exame. Para isso, todas as mulheres responderam um formulário específico de auto-resposta investigando os aspectos acima citados e foram submetidas a avaliação antropométrica da face, avaliação dento-oclusal, avaliação perceptivo-auditiva da voz, bem como avaliação da linguagem expressiva e receptiva.

Para o exame da fonetografia foi utilizado o Programa VRP (Voice Range Profile) da Multi Speech, da Kay Elemetrics ${ }^{\circledR}$, com um microfone de cabeça unidirecional, marca RM MZ3R posicionado a uma distância de $3 \mathrm{~cm}$ da comissura labial do participante. As gravações foram realizadas em sala acusticamente tratada. Para a obtenção das medidas da fonetografia foi solicitada a emissão da vogal "a" prolongada em registro modal, por no mínimo cinco segundos, nas intensidades mais forte e mais fraca possível. O teste foi iniciado em $\mathrm{C}_{4}\left(\mathrm{do}_{4}, 262 \mathrm{~Hz}\right)$ para as participantes, em escala ascendente e posteriormente descendente.

As medidas analisadas foram:

- frequência fundamental mínima e frequência fundamental máxima da voz $\left(\mathrm{F}_{\text {MÍN }}\right.$ e $\left.\mathrm{F}_{\text {MÁX }}\right)$, expressas em semitons e em
Hertz $(\mathrm{Hz})$, que correspondem respectivamente às notas mais agudas e mais graves da escala musical produzidas nas intensidades forte e fraca;

- extensão vocal (EV), expressa em semitons e em Hz, que é a extensão da frequência, compreendendo o número total de notas musicais reproduzidas e é analisada pela diferença existente entre frequência máxima e mínima;

intensidade mínima e intensidade máxima $\left(\mathrm{I}_{\mathrm{MÍN}}\right.$ e $\left.\mathrm{I}_{\mathrm{MÁX}}\right)$ obtidas a partir do ponto mais baixo da curva inferior e do ponto mais alto da curva superior do fonetograma;

- extensão dinâmica máxima (EDM) expressa em decibels (dB), considerada como sendo a maior diferença de intensidade existente entre as curvas superior e inferior do fonetograma em uma mesma frequência;

- área do fonetograma, área resultante da conexão entre todos os pontos da curva inferior e superior em relação à extensão vocal, expressa em dB.st e $\mathrm{cm}^{2}$. Foi obtida a partir dos dados do VRP, por meio de um programa ${ }^{(14)}$ que realiza a conversão de $\mathrm{dB}$.st para $\mathrm{cm}^{2}$, utilizando o fator de conversão de $0,045 \mathrm{~cm}^{2}$, onde $10 \mathrm{~dB}$ correspondem a $15 \mathrm{~mm}$ e 12 st correspondem à $36 \mathrm{~mm}^{(3)}$.

As medidas de todas as frequências apresentadas na abscissa do fonetograma obtidas pelo VRP na fonetografia (Figura 1) foram convertidas de Hertz para semitom, a fim de permitir a realização do cálculo da área do fonetograma, por ser o semitom uma medida linear e apresentar intervalos regulares em escala musical de 12 semitons, o que não ocorre com o Hz, que é uma medida exponencial. Além disso, os valores de extensão vocal na literatura vêm sendo utilizados em semitons $^{(4,7,9,10,15-18)}$, possibilitando comparações posteriores.

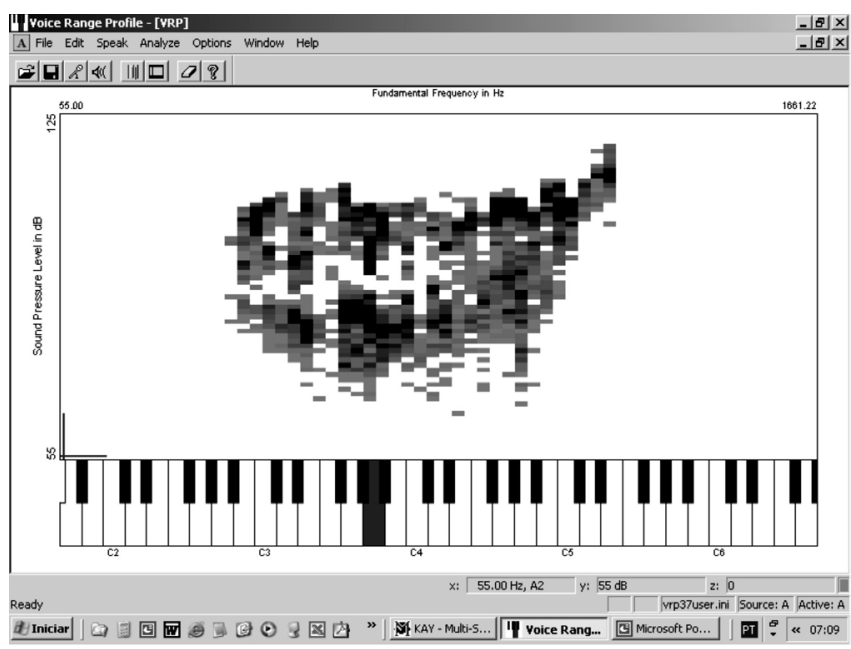

Figura 1. Tela do programa Voice Range Profile da Kay Elemetrics $®$, com o fonetograma computadorizado representando as intensidades mínimas e máximas por toda a extensão de frequência vocal de um indivíduo

Para a análise da frequência fundamental habitual as vozes das participantes foram gravadas em computador por meio do programa Sound Forge 7.0, utilizando o microfone de cabeça unidirecional AKG 444, na posição sentada. A amostra de fala gravada foi a vogal "a" sustentada em tom e intensidade habituais, analisada no programa Multi Dimensional Voice Program (MDVP) da Kay Elemetrics®. 
Para demonstrar os resultados, foi utilizada análise estatística descritiva.

\section{RESULTADOS}

A partir do exame de fonetografia, foram encontradas as medidas de frequência fundamental mínima e frequência fundamental máxima, e o número de semitons da extensão vocal. Foram analisados, ainda, os valores da frequência fundamental habitual $\left(\mathrm{F}_{0}\right)$ da vogal "a" sustentada das 22 participantes que realizaram a fonetografia. Tais informações encontram-se apresentadas na Tabela 1 .

Tabela 1. Valores referentes à frequência fundamental habitual e extensão vocal dos participantes

\begin{tabular}{|c|c|c|c|c|c|c|}
\hline \multirow[t]{3}{*}{ Sujeito } & \multicolumn{5}{|c|}{ Frequência fundamental } & \multirow{3}{*}{$\begin{array}{c}\begin{array}{c}\text { Extensão } \\
\text { vocal }\end{array} \\
\text { (st) }\end{array}$} \\
\hline & \multicolumn{2}{|c|}{ Mínima } & \multicolumn{2}{|c|}{ Máxima } & \multirow{2}{*}{$\begin{array}{c}\text { Habitual } \\
(\mathrm{Hz})\end{array}$} & \\
\hline & $(\mathrm{Hz})$ & (st) & $(\mathrm{Hz})$ & (st) & & \\
\hline 1 & 185 & 42 & 698 & 65 & 215 & 23 \\
\hline 2 & 156 & 39 & 622 & 63 & 185 & 24 \\
\hline 3 & 175 & 41 & 784 & 67 & 229 & 26 \\
\hline 4 & 185 & 42 & 1109 & 73 & 223 & 31 \\
\hline 5 & 139 & 37 & 880 & 69 & 210 & 32 \\
\hline 6 & 156 & 39 & 932 & 70 & 243 & 31 \\
\hline 7 & 175 & 41 & 784 & 67 & 221 & 26 \\
\hline 8 & 185 & 42 & 1109 & 73 & 193 & 31 \\
\hline 9 & 147 & 38 & 523 & 60 & 191 & 22 \\
\hline 10 & 165 & 40 & 880 & 69 & 237 & 29 \\
\hline 11 & 147 & 38 & 831 & 68 & 203 & 30 \\
\hline 12 & 156 & 39 & 831 & 68 & 208 & 29 \\
\hline 13 & 123 & 35 & 622 & 63 & 186 & 28 \\
\hline 14 & 196 & 43 & 740 & 66 & 238 & 23 \\
\hline 15 & 156 & 39 & 1319 & 76 & 212 & 37 \\
\hline 16 & 196 & 43 & 1109 & 73 & 221 & 30 \\
\hline 17 & 165 & 40 & 932 & 70 & 179 & 30 \\
\hline 18 & 185 & 42 & 1047 & 72 & 219 & 30 \\
\hline 19 & 185 & 42 & 880 & 69 & 199 & 27 \\
\hline 20 & 131 & 36 & 988 & 71 & 164 & 35 \\
\hline 21 & 165 & 40 & 1047 & 72 & 256 & 32 \\
\hline 22 & 208 & 44 & 1319 & 76 & 210 & 32 \\
\hline $\bar{x}$ & 167,32 & 40,09 & 908,45 & 69,09 & 211,00 & 29,00 \\
\hline DP & 22,27 & 2,37 & 10,93 & 4,13 & 22,55 & 3,85 \\
\hline
\end{tabular}

Legenda: $\mathrm{Hz}=\mathrm{Hertz} ; \mathrm{st}=$ semitom; $\overline{\mathrm{X}}=$ média; $\mathrm{DP}=$ desvio-padrão

Foram obtidos os valores da fonetografia relacionados à intensidade mínima, intensidade máxima, e à EDM, bem como os valores da área do fonetograma, apresentados na Tabela 2.

\section{DISCUSSÃO}

A fonetografia é capaz de registrar todas as possibilidades laríngeas em relação à frequência e à intensidade sonora, uma vez que abrange os sons mais agudos e mais graves que a
Tabela 2. Valores da intensidade, extensão dinâmica máxima e da área total do fonetograma

\begin{tabular}{|c|c|c|c|c|c|}
\hline \multirow[t]{2}{*}{ Sujeito } & \multicolumn{2}{|c|}{ Intensidade (dB) } & \multirow[t]{2}{*}{ EDM (dB) } & \multicolumn{2}{|c|}{ Área } \\
\hline & Mínima & Máxima & & (dB.st) & $\left(\mathrm{cm}^{2}\right)$ \\
\hline 1 & 72 & 106 & 28 & 440,5 & 19,8 \\
\hline 2 & 69 & 100 & 27 & 527,0 & 23,7 \\
\hline 3 & 72 & 109 & 30 & 484,5 & 21,8 \\
\hline 4 & 69 & 110 & 30 & 638,5 & 28,7 \\
\hline 5 & 76 & 111 & 27 & 654,5 & 29,5 \\
\hline 6 & 76 & 117 & 31 & 531,5 & 23,9 \\
\hline 7 & 72 & 105 & 27 & 458,5 & 20,6 \\
\hline 8 & 71 & 122 & 38 & 851,5 & 38,3 \\
\hline 9 & 69 & 112 & 36 & 568,0 & 25,6 \\
\hline 10 & 65 & 119 & 47 & 885,5 & 39,8 \\
\hline 11 & 68 & 121 & 35 & 666,5 & 30,0 \\
\hline 12 & 71 & 117 & 43 & 944,5 & 42,5 \\
\hline 13 & 68 & 106 & 34 & 630,0 & 28,4 \\
\hline 14 & 65 & 109 & 31 & 528,0 & 23,8 \\
\hline 15 & 66 & 112 & 36 & 975,5 & 43,9 \\
\hline 16 & 70 & 117 & 39 & 791,5 & 35,6 \\
\hline 17 & 72 & 122 & 38 & 797,0 & 35,9 \\
\hline 18 & 69 & 116 & 34 & 741,5 & 33,4 \\
\hline 19 & 73 & 116 & 34 & 658,5 & 29,6 \\
\hline 20 & 71 & 121 & 42 & 942,5 & 42,4 \\
\hline 21 & 61 & 111 & 42 & 845,5 & 38,0 \\
\hline 22 & 60 & 110 & 39 & 1048,0 & 47,2 \\
\hline $\bar{x}$ & 69,32 & 113,14 & 34,91 & 709,50 & 31,93 \\
\hline DP & $\pm 4,07$ & $\pm 6,10$ & $\pm 5,71$ & $\pm 182,92$ & $\pm 8,23$ \\
\hline
\end{tabular}

Legenda: $\bar{X}$ = média; $\mathrm{DP}=$ desvio-padrão; $\mathrm{dB}=$ decibel; $\mathrm{EDM}=$ extensão dinâmica máxima

laringe humana é capaz de produzir e as maiores e menores intensidades envolvidas na emissão de uma vogal ${ }^{(3,4,7,19)}$. Poucos são os estudos voltados para a obtenção de parâmetros de normalidade para falantes do Português Brasileiro.

Neste estudo, as medidas da frequência e intensidade foram analisadas nos extremos da extensão vocal para mulheres adultas jovens, com equilíbrio dentofacial e voz normal, por meio da fonetografia computadorizada.

Com relação às médias da frequência mínima e da frequência máxima do fonetograma, os resultados mostraram-se semelhantes aos obtidos para um grupo controle composto por mulheres de 18 a 65 anos, cujos autores compararam indivíduos saudáveis com pessoas com refluxo ${ }^{(16)}$, sendo importante considerar a distinção entre a faixa etária dos estudos. Ao compararmos com os valores obtidos para mulheres com pólipos nas pregas vocais, de idade entre 18 e 67 anos, os valores médios do presente estudo foram numericamente maiores, o que pode estar relacionado a deteriorizacao da qualidade e capacidade vocal decorrente da irregularidade da vibração e desequilíbrio da massa entre as pregas vocais destes indivíduos $^{(20)}$. Comparando-se com um estudo de mulheres idosas, os valores do presente trabalho também foram maiores ${ }^{(4)}, \mathrm{o}$ 
que pode ter sido influenciado pela idade das participantes, uma vez que essa é uma fase de intensa mudança hormonal e consequentemente na voz, como mostrado em alguns estudos que afirmam que o perfil vocal de cada indivíduo depende das características anatômicas, fisiológicas e dos fatores hormonais $^{(6,21)}$.

A média da extensão vocal obtida nesse estudo foi maior do que os resultados apresentados em um estudo de mulheres jovens ${ }^{(10)}$ e também de mulheres com idades entre 41 e 60 $\operatorname{anos}^{(9)}$. Isso pode ser justificado pela diminuição da secreção de hormônios estrógenos, no período da pós-menopausa, resultando em alterações morfológicas na laringe e, consequentemente, em diminuição das frequências ${ }^{(8)}$. Além disso, um edema de pregas vocais nas mulheres pode ser causado por mudanças endócrinas após a menopausa ${ }^{(22)}$.

Com relação à $\mathrm{F}_{0}$ para a vogal /a/ sustentada, ao compararmos o valor médio encontrado com os resultados obtidos em um estudo que também investigou a medida da $F_{0}$ em mulheres adultas falantes do Português Brasileiro, os achados foram semelhantes ${ }^{(23)}$.

Considerando os valores médios obtidos quanto às intensidades mínima e máxima, ao compará-los com trabalhos já citados, os resultados encontrados no presente estudo foram numericamente maiores ${ }^{(4,19)}$. Tais achados podem indicar maior pressão aérea à fonação, bem como, maior controle laríngeo à passagem da corrente aérea pelos sujeitos do presente estudo ${ }^{(24)}$.

A média da EDM foi semelhante aos valores encontrados em um estudo de vozes de mulheres adultas jovens ${ }^{(10)}$ e em mulheres com idades variando entre 41 e 60 anos $^{(9)}$. Porém, a média da EDM de mulheres acima de 60 anos $^{(4)}$, foi menor do que a encontrada em mulheres mais jovens. Pode-se inferir que essa redução parece estar relacionada ao processo normal do envelhecimento, como fraqueza nos músculos da laringe, mudança no esforço respiratório e diminuição no contato das pregas vocais ${ }^{(25)}$.

Quanto à medida da área do fonetograma, o valor médio encontrado neste estudo foi semelhante ao relatado para mulheres do grupo controle no trabalho que comparou indivíduos saudáveis com indivíduos com refluxo laríngeo ${ }^{(16)}$ porém, maior do que o obtido em mulheres com pólipos nas pregas vocais $^{(20)}$, em adultas jovens ${ }^{(10)}$ e em mulheres entre 40 e 60 $\operatorname{anos}^{(9)}$. Fica evidente que a área do fonetograma apresentouse maior, quando comparada a esses estudos citados, uma vez que nesse trabalho houve também maiores valores de extensão vocal, repercutindo nos valores da área do gráfico.

Apesar da literatura apresentada não considerar a condição dentofacial dos indivíduos, os resultados obtidos foram semelhantes em relação à $\mathrm{EDM}^{(9,10)} \mathrm{eF}_{0}^{(23)}$, sugerindo que os aspectos anatômicos do sistema estomatognático não influenciam tais parâmetros acústicos vocais. Por outro lado, de acordo com os achados desse trabalho, a frequência mínima e máxima, a extensão vocal, as intensidades mínima e máxima, assim como a área do fonetograma podem ser influenciados pela posição dos articuladores, os quais apresentam intrínseca relação com a função fonatória da laringe. Além disso, esta pesquisa controlou as variáveis de relação facial e dento-oclusal e, ainda, o período menstrual das participantes, o que também pode justificar os parâmetros acústicos vocais que diferiram da literatura.

Sugere-se que a partir deste estudo, outros trabalhos possam dar continuidade à investigação dos parâmetros vocais, por meio da fonetografia, levando em consideração as diversas tipologias faciais e relação dento-oclusal em vozes normais, a fim de estabelecer possíveis relações.

\section{CONCLUSÃO}

O presente estudo possibilitou verificar o perfil da extensão vocal de mulheres com equilíbrio dentofacial. Os resultados obtidos foram semelhantes aos da literatura, com exceção da área do fonetograma e valores de extensão vocal.

\section{AGRADECIMENTOS}

Agradecemos ao Programa Institucional de Bolsas de Iniciação Cientifica (PIBIC/CNPq), pelo apoio concedido para realização dessa pesquisa, sob processo número 024/2005.

\begin{abstract}
Purpose: To characterize the vocal extension profile of women with dentofacial balance using phonetography. Methods: Took part of this study 22 female students with ages between 18 and 29 years $(\bar{X}=20 \pm 2$ years), all Brazilian Portuguese speakers, without vocal disorders or vocal training, who presented balanced face pattern I and dental-occlusal harmony. The subjects answered a specific self-response form, and were submitted to anthropometric assessment of the face, dental-occlusal evaluation, phonetography examination, and acoustic analysis of the usual fundamental frequency of the participants' voices, carried out in the post-menstrual period. Results: The values found for the different parameters analyzed were: minimum fundamental frequency: $\overline{\mathrm{X}}=167,32 \pm 22,27$ $\mathrm{Hz}$ or $\overline{\mathrm{X}}=40,09 \pm 2,37 \mathrm{st}$, maximum fundamental frequency: $\overline{\mathrm{X}}=908,45 \pm 210,93 \mathrm{~Hz}$ or $\overline{\mathrm{X}}=69,09 \pm 4,13$ st, voice extension: $\bar{X}=29,00 \pm 3,85$ st, minimum intensity: $\bar{X}=69,32 \pm 4,07 \mathrm{~dB}$, maximum intensity: $\bar{X}=113,14 \pm 6,10 \mathrm{~dB}$, maximum dynamic extension: $\bar{X}=3,91 \pm 5,71 \mathrm{~dB}$, phonetogram area: $\bar{X}=709,50 \pm 182,92 \mathrm{~dB} \cdot \mathrm{st}$ ou $\bar{X}=31,93 \pm 8,23 \mathrm{~cm}^{2}$, usual fundamental frequency of the vowel "a": $\bar{X}=211,00 \pm 22,55 \mathrm{~Hz}$. Conclusion: The present study obtained the minimum and maximum intensity levels for different frequencies achieved by the participants, as well as the usual fundamental frequency, establishing reference values for the vocal extension profile of Brazilian woman without voice disorders and with dentofacial balance.
\end{abstract}

Keywords: Voice/physiology; Fonation/physiology; Voice quality; Speech acoustics; Face; Stomatognathic system 


\section{REFERÊNCIAS}

1. DeJonckere PH, van Wijck I, Speyer R. Efficacy of voice therapy assessed with the Voice Range Profile (Phonetogram). Rev Laryngol Otol Rhinol (Bord). 2003;124(5):285-9.

2. Damsté PH. The phonetogram. Pract Otorhinolaryngol (Basel). 1970;32(3):185-7.

3. Schutte HK, Seidner W. Recommendation by the Union of European Phoniatricians (UEP): standardizing voice area measurement/ phonetography. Folia Phoniatr (Basel). 1983;35(6):286-8.

4. Teles-Magalhaes LC, Pegoraro-Krook MI, Pegoraro R. Study of the elderly females' voice by phonetography. J Voice. 2000;14(3):310-21.

5. Dmitriev L, Kiselev A. Relationship between the formant structure of different types of singing voices and the dimensions of supraglottic cavities. Folia Phoniatr (Basel). 1979;31(4):238-41.

6. Oliveira VL, Pinho SMR. A qualidade da voz e o trato vocal nos indivíduos de face curta e face longa. In: Pinho SMR. Tópicos em voz. Rio de Janeiro: Guanabara Koogan; c2001. p. 81-8.

7. Wuyts FL, Heylen L, Mertens F, Du Caju M, Rooman R, Van de Heyning PH, De Bodt M. Effects of age, sex, and disorder on voice range profile characteristics of 230 children. Ann Otol Rhinol Laryngol. 2003;112(6):540-8.

8. Nishio M, Niimi S. Changes in speaking fundamental frequency characteristics with aging. Folia Phoniatr Logop. 2008;60(3):120-7.

9. Teixeira MT, Pegoraro-Krook MI, Marino VCC, Pegoraro R. Perfil da extensão vocal (fonetografia) em mulheres com vozes normais. In: Encontro científico da pós-graduação do HPRLLP-USP, Bauru - SP, 1997. Anais. Bauru: HPRLLP-USP; 1997. p. 34.

10. Marino VCC. Perfil da extensão vocal em vozes normais no sexo feminino [dissertação]. São Paulo: Universidade Federal de São Paulo. Escola Paulista de Medicina; 1995.

11. Reis SAB, Abrão J, Capelozza Filho L, Claro CAA. Análise facial numérica do perfil de brasileiros Padrão I. Rev Dent Press Ortodon Ortopedi Facial. 2006;11(6):24-34.

12. Angle EH. Classification of malocclusion. Dental Cosmos. 1899;41(3):248-64.

13. Langlade M. Diagnóstico ortodôntico. São Paulo: Santos; 1995.
14. Magalhães MK. Banco de dados sobre fonetografia e elaboração digital do fonetograma [dissertação]. São Carlos: Bioengenharia da Universidade de São Paulo; 2004.

15. Titze IR, Wong D, Milder MA, Hensley SR, Ramig LO. Comparison between clinician-assisted and fully automated procedures for obtaining a voice range profile. J Speech Hear Res. 1995;38(3):526-35.

16. Pribuisiene R, Uloza V, Saferis V. Multidimensional voice analysis of reflux laryngitis patients. Eur Arch Otorhinolaryngol. 2005;262(1):3540.

17. Ma E, Robertson J, Radford C, Vagne S, El-Halabi R, Yiu E. Reliability of speaking and maximum voice range measures in screening for dysphonia. J Voice. 2006;21(4):397-406.

18. Pribuisiene R, Uloza V, Kupcinskas L, Jonaitis L. Perceptual and acoustic characteristics of voice changes in reflux laryngitis patients. $\mathrm{J}$ Voice. 2006;20(1):128-36.

19. Camargo TF, Barbosa DA, Teles LCS. Características da fonetografia em coristas de diferentes classificações vocais. Rev Soc Bras Fonoaudiol. 2007;12(1):10-7.

20. Uloza V, Kaseta M, Pribuisiene R, Saferis V, Jokūzis V, Gelzinis A, Bacauskiene M. Quantitative microlaryngoscopic measurements of vocal fold polyps, glottal gap and their relation to vocal function. Medicina (Kaunas). 2008;44(4):266-72.

21. Quinteiro EA. Higiene da voz. In: Quinteiro EA. Estética da voz: uma voz para o ator. São Paulo: Summus; 1989. p. 70-6.

22. Honjo I, Isshiki N. Laryngoscopic and voice characteristics of aged persons. Arch Otolaryngol. 1980;106(3):149-50.

23. Araújo SA, Grellet M, Pereira JC, Rosa MO. Normatização de medidas acústicas da voz normal. Rev Bras Otorrinolaringol. 2002;68(4):540-4.

24. Behlau M, Dragone MLS, Nagano L. Voz saudável e disfonia. In: Behlau M, Dragone MLS, Nagano L. A voz que ensina: o professor e a comunicação oral em sala de aula. Rio de Janeiro: Revinter; c2004. p.5-15.

25. Gorham-Rowan MM, Laures-Gore J. Acoustic-perceptual correlates of voice quality in elderly men and women. J Commun Disord. 2006;39(3):171-84. 